\title{
Contact electrification and charge distribution on elongated particles in a vibrating container
}

Chunlei Pei, ${ }^{1,2}$ Chuan-Yu Wu, ${ }^{2 *}$ Michael Adams, ${ }^{1}$ David England, ${ }^{3}$ Stephen Byard ${ }^{4}$ and Harald Berchtold ${ }^{5}$

${ }^{1}$ School of Chemical Engineering, University of Birmingham, Birmingham, B15 2TT, UK

${ }^{2}$ Department of chemical and process engineering, University of Surrey, Guildford, Surrey, GU2 7XH, UK

${ }^{3}$ Private consultant, Previously with Sanofi-Aventis UK

${ }^{4}$ Covance Laboratories, Alnwick, Northumberland NE66 2JH, UK

${ }^{5}$ Sanofi-Aventis Deutschland GmbH, Frankfurt, Germany

\begin{abstract}
The electrostatic charge can be transferred between particles during collisions. The particle shape plays an important role and, in the current study, the charge accumulation and distribution on elongated particles in a vibrating container are investigated using a discrete element method in which a contact electrification model is implemented. The elongated particle geometry is modelled using a multi-sphere approach. Five different shapes are considered and characterized using a shape factor, $\delta$, which is defined as the ratio of the difference of the radii between the distal sphere and central sphere to the mean radius of the particle. It is found that the net charge on the central sphere is greater than that on the distal sphere when $\delta<0$. For a particle with $\delta>0$, greater net charge is accumulated on the larger distal sphere. The maximum surface charge difference between the distal and central sphere increases as the shape factor increases. The net charge of the granular system with different particle shapes achieves an equilibrium state during the vibrating process. This accumulating process follows an exponential trend.
\end{abstract}

Keywords: contact electrification; electrostatics; irregular particles; discrete element method; multi-sphere approach

\section{Introduction}

Contact electrification is a charge transfer process between objects during collisions in powder handling processes. The contact potential difference, which is considered as the difference in electron affinities, is the driving force during contact electrification so that the charge is transferred from one surface to another during contact (Matsusaka et al., 2000, 2010). However, for highly insulating materials, the transferred charge can be retained on particle surfaces due to slow electrostatic relaxation and redistribution (Haelen, 1976; Kornfeld, 1976), which will lead to a non-uniform charge distribution on particles. With the accumulated charge, the induced electrostatic force can become dominant and lead to undesirable phenomena, such as particle aggregation and segregation (Grzybowski et al., 2003; Pei et al., 2010; Nwose et al., 2012). Therefore, the study of charge accumulation and distribution on particles is important for a more detailed understanding of particle dynamics in powder handling processes.

\footnotetext{
* Corresponding Author. Tel: 0044-1483 68 3506; Fax: 0044-1483 68 6581. Email: C.Y.Wu@surrey.ac.uk
} 
Surface charge distribution on insulating materials was examined experimentally, by Liu and Bard (2009) and Rezende et al. (2009). Liu and Bard (2009) used a corner of a piece of poly(methylmethacrylate) (PAMM) as a pen to rub on the surface of polytetrafluoroethylene (PTFE) with a predefined pattern (Chinese characters). Then graphite powder was used to decorate the surface of the PTFE. It was observed that the powder was only attracted and located in the rubbed area and made the predefined pattern visible. This indicates a nonuniform distribution of charge on the surface of the insulating material. The transferred charge on the surface of the PTFE can be stable in the contact area over many minutes. Rezende et al. (2009) reviewed several experimental measurement methods for special charge detection on insulator surfaces and found that the charge distribution on the surface of insulators can show complex and different patterns. For example, Kelvin force microscopy (KFM) was used to obtain the electric potential distribution (image) of poly(styrene-cohydroxyethyl methacrylate) (PS-HEMA) dry latex. It is found that, due to the anisotropic electrical polarity of the particle, the electric potential on the surface of the particle was not uniformly distributed and there was excess concentrated charge on the particle. However, if the charge concentration is relatively small $\left(<10^{-10} \mathrm{~mol} / \mathrm{l}\right)$, it is still difficult to quantify and analyze the charge density and distribution even with some sensitive methods, such as the analytic transmission electron microscopy method (Rezende et al., 2009).

Particle shape plays an important role in charge transfer during contact. Watanabe et al. (2007) investigated the contact electrification of various pharmaceutical particles impacting a tilted steel surface and found that, assuming the particle is spherical, the transferred charge is generally linearly proportional to the estimated contact area. However, ethlycellulose particles developed a different transferred charge from that calculated by assuming a spherical particle shape. It was concluded that the irregular shape of an ethlycellulose particle might result in sliding or rolling after impact, which would change the contact area and the charge transfer process. Ireland (2010a, 2010b) observed different modes of contact, including sliding (contact), rolling and bouncing when particles were dropped on a titled surface. Ireland $(2010 \mathrm{~b}, 2012)$ argued that the irregular shape of a particle could affect the modes of contact and the contact area and subsequently the charge transfer and distribution during contact.

It is still difficult to apply the above experimental methods during powder handing processes in order to determine the surface charge distribution concurrently. Therefore, numerical methods have also been employed to understand the electrostatic charge distribution on a particle surface in a granular system during collisions (Duff and Lacks, 2008; Liu et al., 2010; Ireland, 2012). Duff and Lacks (2008) randomly generated points on the surface of spherical particles and assumed that high energy electrons were trapped at these points. A hard-sphere model was then used to simulate the motion of the particles with initial random velocities. When the points on a given particle were within the contact region, the trapped electrons would be transferred to area region with the lowest energy state on the contacting particle. Although the initial surface charge density was identical for particles with different sizes, it was shown that larger particles were charged positively while smaller particles became negatively charged. Although this method utilised the randomly generated points with high energy electrons to represent the charge distribution on the surface of a particle, it was not able to accurately analyse the charge transfer and the charge distribution during collisions.

In order to determine the charge distribution, the particle surface is usually divided into meshes or elements so that the charge concentration in each element could be determined and subsequently the charge distribution could be obtained. Liu et al. (2010) discretized the 
surfaces of spherical particles and a cylindrical electrode into meshes and used a boundary element method (BEM) to simulate the charge distribution on the surface of the particles induced by an electric field. A discrete element method (DEM) was applied to simulate the dynamics of the particles in the electric field. The induced electrostatic interactions forced particles to deposit on the cylindrical electrode and form straight particle chains. This method can be used to calculate the electrostatic interactions and model the surface charge distribution for conductors and dielectrics. However, in order to determine the surface charge generated by contact electrification within a collisional system, this method can be extremely computationally intensive as finer meshes are required to detect the contact region especially when 3D irregularly shaped particles are considered. Ireland (2012) modelled the charge transfer between a 2D elliptical particle and a tilted surface during impact using DEM. The surface of the particle was discretized into segments and the charge was only transferred onto the segments inside the contact region because of the resulting nature of the particle. It was shown that, when the particle made contact with the surface, a smaller roundness ratio, which was defined as the ratio of radii between the major and the minor axis, lead to a larger contact area. And also the transferred charge was greater with a larger contact area, which meant that the charge transfer process could be affected by the particle shape.

In the current study, a 3D discrete element model for contact electrification of irregular shaped particles is developed. The charging process and charge distribution on particles in a vertically vibrating container are modelled. The contact frequency at different parts of the particle is further examined to explore its relationship with the charge distribution on the particles.

\section{The DEM Model}

Using a current DEM computer programme (Kafui et al., 2002), elongated particle shapes were approximated using the symmetric multi-sphere model (Favier et al., 1999) in which a particle is assembled by a row of primary spheres of various sizes with negligible overlaps (i.e. $<5 \%$ of the sphere radius). For each particle, the primary spheres are rigidly connected without relative movement. Thus the mass and moment of inertia of the particle can be calculated as a summation of all the primary spheres. Contact detection and contact force calculation are based on the primary spheres. The contact of particles is detected between constituent primary spheres as shown in Figure 1. 


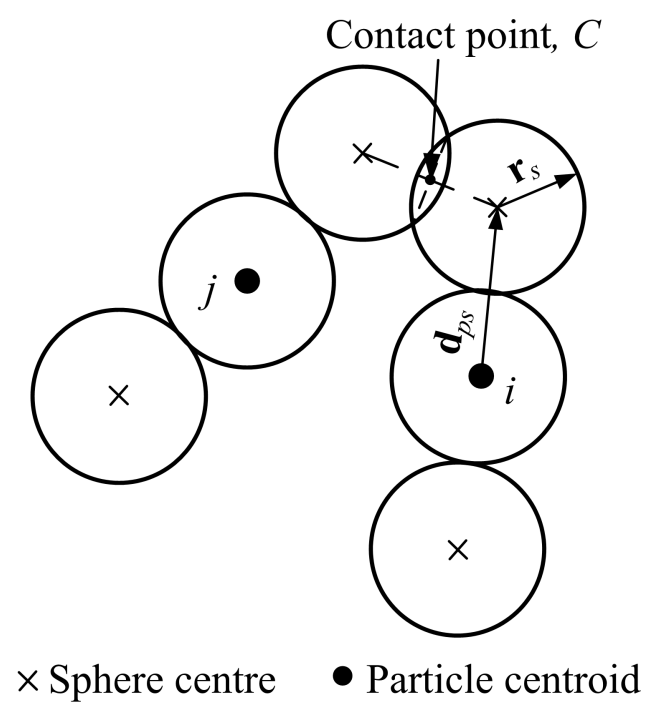

Figure 1 Contact detection between constituent primary spheres of multi-sphere particles where $\mathrm{x}$ and $\mathrm{o}$ correspond to the centres of each primary sphere and the centroids of the particles.

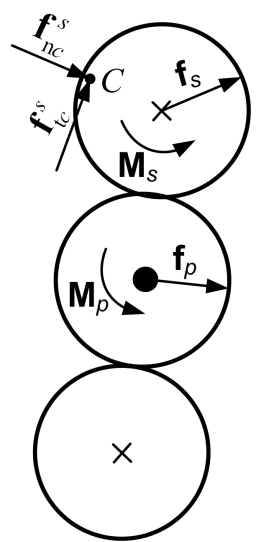

Figure 2 Forces acting on the multi-sphere particle $i$ shown in Figure 1.

Contact forces and moments between primary spheres of different particles are calculated once the contacts between these primary spheres are determined, as illustrated in Figure 2. For elastic particles, the normal contact is modelled using Hertz theory (Johnson, 1985), and that of Mindlin and Deresiewicz (1953) is employed for the tangential interaction. The contact forces and moments are first summed on each primary sphere, $s$, as:

$$
\begin{aligned}
& \mathbf{f}_{s}=\sum_{c=1}^{c_{n}}\left(\mathbf{f}_{\mathrm{n} c}^{s}+\mathbf{f}_{\mathrm{t} c}^{s}\right) \\
& \mathbf{M}_{s}=\sum_{c=1}^{c_{n}}\left(\mathbf{r}_{s c} \times \mathbf{f}_{\mathrm{t} c}^{s}\right)
\end{aligned}
$$

where, $\mathbf{f}_{\mathrm{n} c}^{s}, \mathbf{f}_{\mathrm{tc}}^{s}$ are the normal and tangential contact force at the contact point $c ; \mathbf{r}_{s c}$ is the vector from the centre of the primary sphere to the contact point $c ; c_{n}$ is the total number of contacts on the primary sphere; $\mathbf{f}_{s}, \mathbf{M}_{s}$ are the resultant force and moment. 
As the primary spheres are rigidly connected, the force and toque acting on each primary sphere are then summed onto the particle centroid as (Favier et al., 1999):

$$
\begin{gathered}
\mathbf{f}_{p}=\sum_{s=1}^{n_{p}} \mathbf{f}_{s} \\
\mathbf{M}_{p}=\sum_{s=1}^{n_{p}}\left(\mathbf{d}_{s} \times \mathbf{f}_{s}+\mathbf{M}_{s}\right)
\end{gathered}
$$

where, $\mathbf{d}_{s}$ is the vector from the particle centroid to the centre of the primary sphere $\mathrm{s} ; n_{p}$ is the total number of primary spheres of the particle $p ; \mathbf{f}_{p}, \mathbf{M}_{p}$ are the resultant force and moment of the particle. The subsequent translational and rotational motion of the particle are determined using Newton's second law.

The contact electrification model (Pei et al., 2013) was implemented to analyze the charge transfer process during collisions. According to the condenser model, the charge transfer is due to the total potential difference, $\Delta V$, as follows.

$$
\Delta V=V_{c}-V^{\prime}=V_{i}-V_{j}-V^{\prime}
$$

where $V_{c}\left(=V_{i}-V_{j}\right)$ is the contact potential difference (CPD) between the surfaces; $V$ is the induced potential difference; $V_{i}$ and $V_{j}$ are the work function potentials of particles $i$ and $j$, respectively.

For multi-spheres, the charge transfer process is based on the primary spheres in this study. The induced potential difference $V^{\prime}$ is assumed to be only affected by the charge on the primary spheres in contact. Therefore, the induced potential difference $V^{\prime}$ between a plane surface and the primary sphere is written as (Matsuyama and Yamamoto, 1995; Pei et al., 2013):

where

$$
V^{\prime}=k_{0} q_{s}=\frac{z}{4 \pi \varepsilon_{0} r_{s}^{2}} q_{s}
$$

$$
k_{0}=\frac{z}{4 \pi \varepsilon_{0} r_{s}^{2}}=\frac{z}{\varepsilon_{0} A_{s}}
$$

where $r_{s}$ and $A_{s}$ are the radius and surface area of the primary sphere, $\varepsilon_{0}$ is the permittivity of a vacuum $\left(8.854 \times 10^{-12} \mathrm{~F} \cdot \mathrm{m}^{-1}\right), z$ is the gap for tunnel relaxation and is generally of the order of a few nanometers to hundreds of nanometers (Lowell and Roseinnes, 1980). The gap $z$ is assumed to be $260 \mathrm{~nm}$ in this study. The induced electric field can further polarize the plane surface and cause image effects. If the image effects are considered, the induced potential difference can be affected by a factor of 1 - 10, depending on the properties of the particle and the plane surface and the contact conditions (Matsuyama and Yamamoto, 1995).

If two charged primary spheres of insulating materials are considered, then the induced potential difference between them at a given separation distance can be determined as: 


$$
V^{\prime}=\frac{z}{4 \pi \varepsilon_{0}}\left(\frac{q_{s j}}{r_{s j}^{2}}-\frac{q_{s i}}{r_{s i}^{2}}\right)
$$

where $q_{s i}$ and $q_{s j}$ are the charges of the primary spheres $i$ and $j ; r_{s i}$ and $r_{s j}$ are the radii of primary spheres, $i$ and $j$. The transferred charge on primary spheres in each contact can then be calculated as (Matsusaka et al., 2000; Pei et al., 2013):

$$
\Delta q=k S \Delta V
$$

where $S$ is the maximum contact area during the collision, $k$ is the charging constant (Matsusaka et al., 2000; Watanabe et al., 2007) and is of the order of $10^{-4} \mathrm{C} \cdot \mathrm{m}^{-2} \cdot \mathrm{V}^{-1}$. After each collision, the charge on materials $i$ and $j$ will hence become $q_{i}-\Delta q$ and $q_{j}+\Delta q$, respectively. The effects of the net charge on remote primary spheres that are not in the contact under consideration are ignored (Matsuyama et al., 2003). In addition, as the particles are assumed to be perfect insulators, each primary sphere will retain the acquired charges and the charge redistribution and relaxation and electrostatic interactions are also ignored. A full analysis of contact electrification and electrostatic interactions will be presented elsewhere (Pei, 2013).

\section{Model setup}

Contact electrification of elongated particles in a vertically vibrating container (Figure 3 ) is analyzed using the DEM model that was developed. Initially, 50 randomly generated particles are deposited onto the base of a cubic container with a side length of $1 \mathrm{~mm}$ until the kinetic energy of the particle system becomes negligible (i.e. maximum velocity $<1 \times 10^{-6} \mathrm{~m} \cdot \mathrm{s}^{-1}$ ). Then the container starts to vibrate in the $y$ direction with a specified velocity profile as:

$$
v_{b}=v_{a} \sin (2 \pi f t)
$$

where $v_{a}$ is the amplitude of the vibration velocity and is set to $1.0 \mathrm{~m} \cdot \mathrm{s}^{-1} ; f$ is the frequency and is assumed to be $500 \mathrm{~Hz}$ in this study. The simulation was terminated once the total charge of the particles reached an equilibrium state.

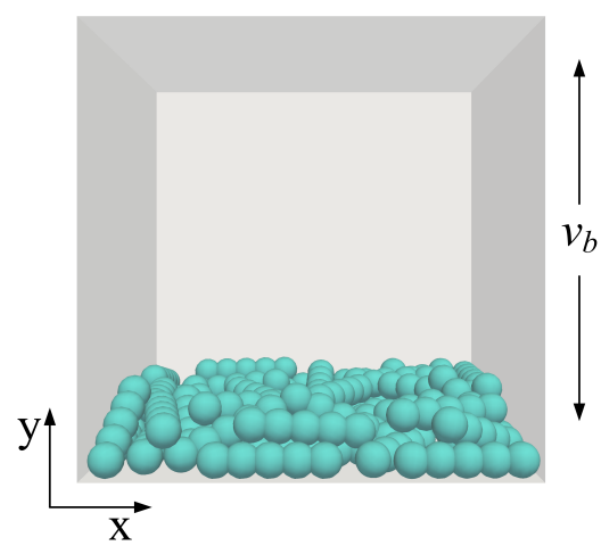

Figure 3 Model setup. 
In this study, the elongated particles are modelled with an array of primary spheres of various sizes. Five types of elongated particles are considered. A shape factor, $\delta$, is introduced to quantify the particle shape and is defined as:

$$
\begin{aligned}
& \delta=\frac{r_{d}-r_{c}}{\bar{r}} \\
& \bar{r}=\frac{1}{n_{g}} \sum_{i=1}^{n_{g}} r_{s i}
\end{aligned}
$$

where $r_{d}$ is the radius of the distal primary sphere, $r_{c}$ is the radius of the central primary sphere, $r_{s i}$ is the radius of the primary sphere $i$ and $n_{g}$ is the number of size types considered, and $\bar{r}$ is the mean radius of each particle.

The shape factor can be used to characterise the concavity of a particle, which is believed to be an important factor in determining the charge distribution during contact electrification. A negative shape factor indicates that the particle has a convex shape profile while a positive value corresponds to a concave profile. The shape factors and the radii of constituent primary spheres for the 5 types of particles considered are given in Table 1, and for each type of particle, 3 sizes $\left(n_{g}=3\right)$ for the primary spheres are considered. The material properties of the primary spheres and the container are given in Table 2. The particles are assumed to be a typical pharmaceutical excipient particles (Watanabe et al., 2007; Nwose et al., 2012) and the container is assumed as stainless steel. The friction coefficients between particles and between the particle and the wall are set to 0.3. The contact damping ratios between particles and between the particle and the wall are 0.016 and 0.032 , respectively, which results in a restitution coefficient in the range of $0.95-0.98$ (Hu et al., 2011). Monosized spherical particles with a radius of $40 \mu \mathrm{m}$ and same material properties are also considered for reference.

The work function potentials of the particles, $V_{p}$, and container, $V_{s}$, are 4.52 and $4.70 \mathrm{~V}$ respectively. The container is assumed to be conductive and the transferred charge will be dissipated instantaneously. The charge accumulation of the particle system and the charge distribution on each particle are analyzed in detail.

Table 1 A list of shaped particles

\begin{tabular}{llll}
\hline Elongated particles & $r_{s i}(\mathrm{i}=1,2,3,4,5)(\mu \mathrm{m})$ & $\bar{r}(\mu \mathrm{m})$ & $\delta$ \\
\hline & $25,40,50,40,25$ & 38.3 & -0.65 \\
& $40,45,50,45,40$ & 45 & -0.22 \\
& $40,40,40,40,40$ & 40 & 0 \\
$50,45,40,45,50$ & 45 & 0.22 \\
& $50,40,25,40,50$ & 38.3 & 0.65 \\
\hline
\end{tabular}


Table 2 Material parameters of the particle and the container.

\begin{tabular}{lll}
\hline & Particle & Container \\
\hline Elastic modulus, $Y(\mathrm{~Pa})$ & $8.7 \times 10^{9}$ & $2.1 \times 10^{11}$ \\
Poisson's ratio, $v$ & 0.3 & 0.3 \\
Density, $\rho\left(\mathrm{kg} \cdot \mathrm{m}^{-3}\right)$ & $1.5 \times 10^{3}$ & $7.9 \times 10^{3}$ \\
\hline
\end{tabular}

To quantify the charge distribution over a particle, the mean charge ratio of the primary spheres with the index $i$ is defined as:

$$
\varphi_{i}=\frac{\bar{q}_{s i}}{\bar{q}}
$$

where $\bar{q}_{s i}$ is the mean charge of the primary spheres with the same index $i ; \bar{q}$ is the mean charge of the particles.

The mean charge variation is defined as the charge difference between a distal primary sphere and a central primary sphere:

$$
q_{v}=\frac{\sum_{i=1}^{N} \Delta q_{s i}}{N}=\frac{\sum_{i=1}^{N}\left(\bar{q}_{d i}-q_{c i}\right)}{N}
$$

where $\bar{q}_{d i}$ is the mean charge of the two distal spheres of particle $i ; q_{c i}$ is the charge of the central sphere of particle $i, N$ is the number of particles.

The surface charge density of a primary sphere can be defined as the charge of the primary sphere divided by its surface area, $\sigma_{s}=q_{s} / A_{s}$, which is used to quantify the surface charge distribution on the particle. In the current study, the mean surface charge difference can be defined as:

$$
\sigma_{v}=\frac{\sum_{i=1}^{N} \Delta \sigma_{s i}}{N}=\frac{\sum_{i=1}^{N}\left(\bar{\sigma}_{d i}-\sigma_{c i}\right)}{N}
$$

where $\bar{\sigma}_{d i}$ is the mean surface charge density of two distal primary spheres of particle $i ; \sigma_{c i}$ is the surface charge density of the central primary sphere of particle $i$.

\section{Results}

\subsection{Particle profiles}

Figure 4 shows a typical charge distribution on each primary sphere during vibration with particles of $\delta=0.0$ at various time instants. The charge acquired during the deposition process is negligible as shown in Figure 4a. The particles vibrate with the container and gradually get charged. During vibration, the charge is accumulated on each primary sphere; the distal spheres generally accumulate more charge than the central ones especially at the 
earlier stage of the vibration (Figure $4 \mathrm{~b}$ and $4 \mathrm{c}$ ). The charge on all primary spheres eventually reaches a maximum value corresponding to an equilibrium state that is reached when all particles possess their equilibrium charges (Figure $4 \mathrm{~d}$ ).

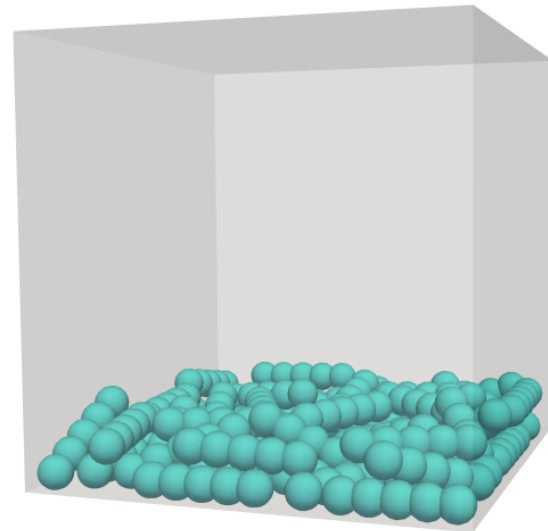

(a) $t=0.0 \mathrm{~s}$

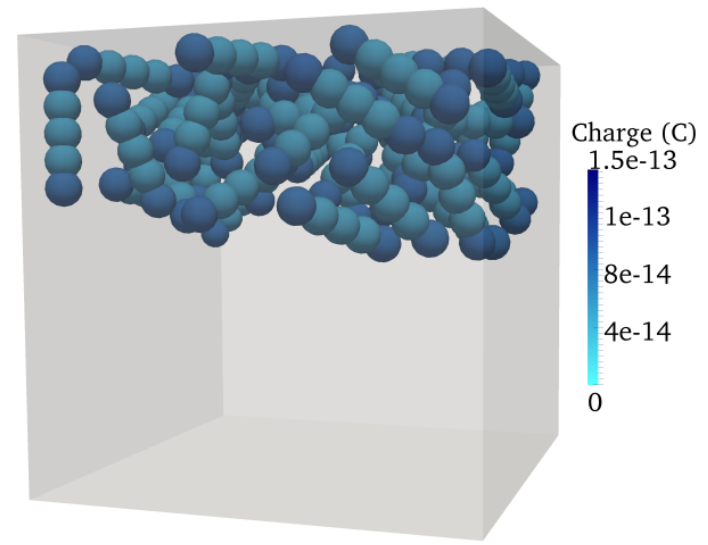

(c) $t=0.54 \mathrm{~s}$

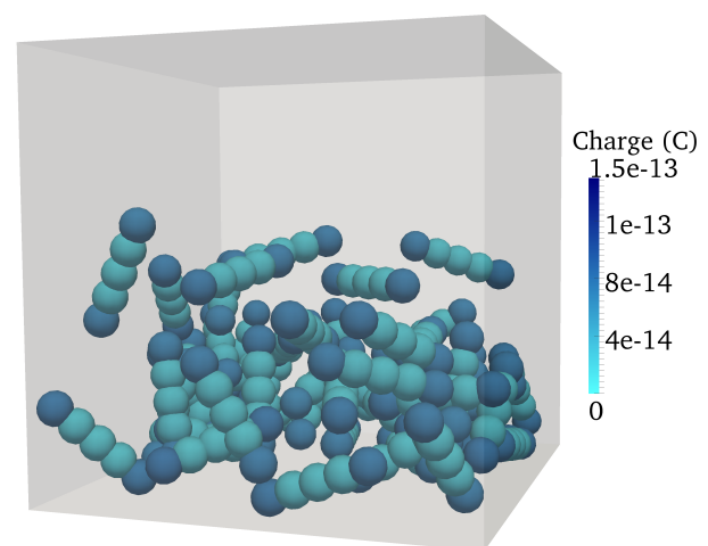

(b) $t=0.27 \mathrm{~s}$

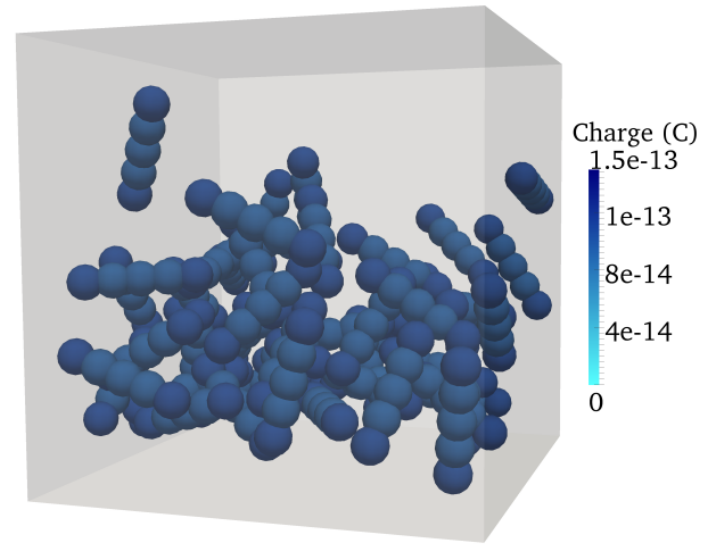

(d) $t=1.08 \mathrm{~s}$

Figure 4 Charge distributions for the particles of $\delta=0.0$ at various time instants.

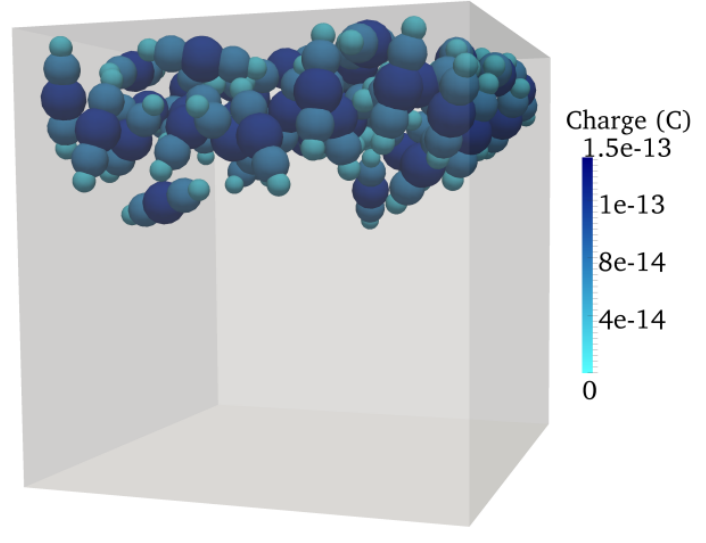

(a) $\delta=-0.65$

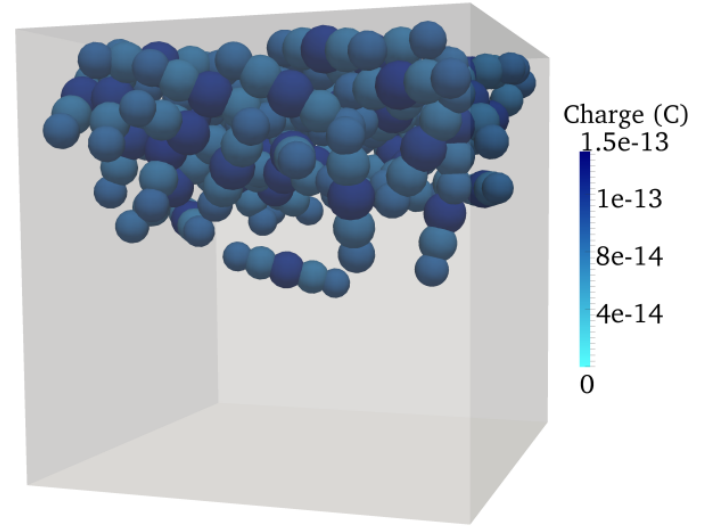

(b) $\delta=-0.22$ 


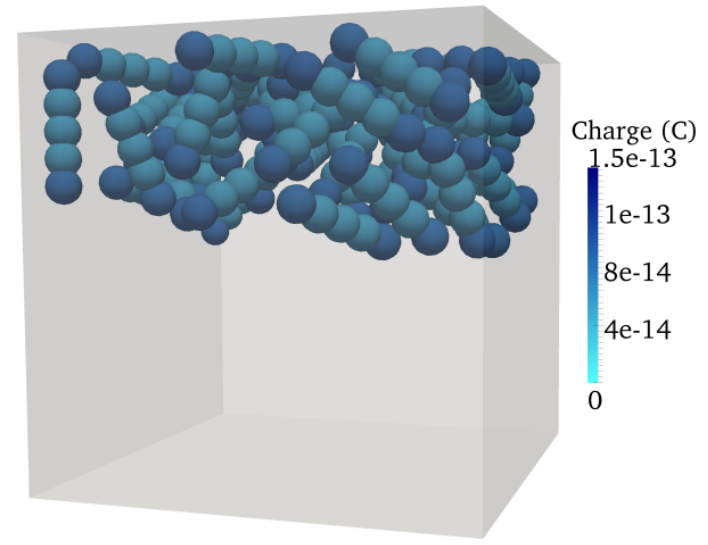

(c) $\delta=0.0$

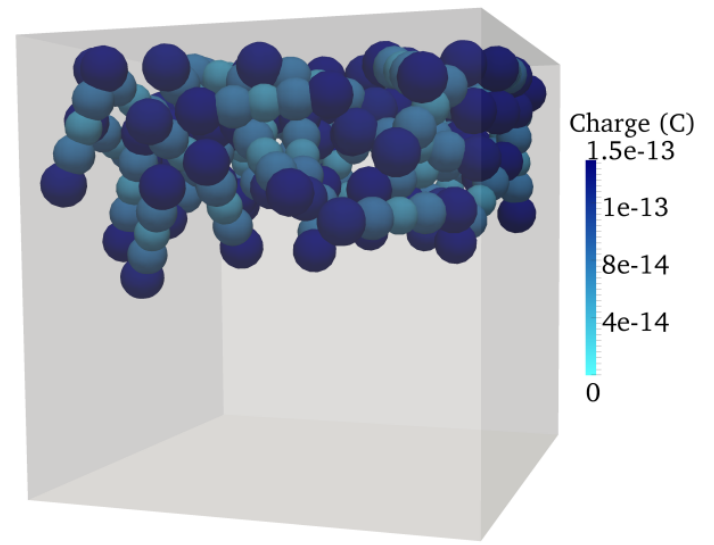

(d) $\delta=0.22$

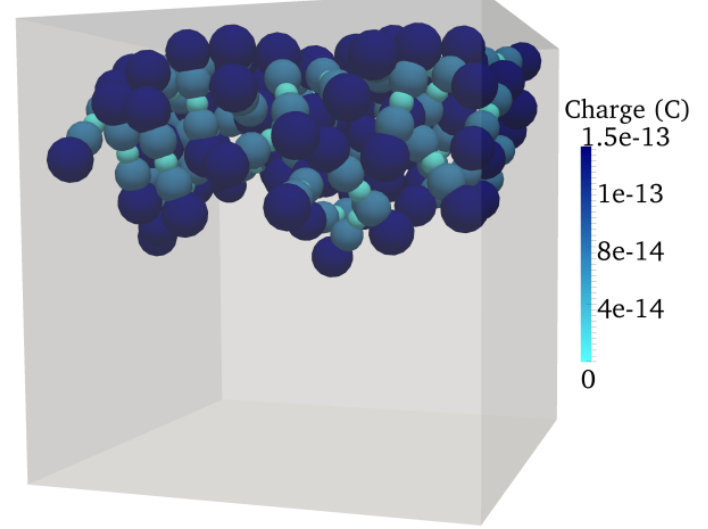

(e) $\delta=0.65$

Figure 5 Charge distributions for various shaped particles at $t=0.54 \mathrm{~s}$.

Figure 5 shows the charge distribution over particles with different shape factors at $t=0.54 \mathrm{~s}$. It is clear that the charge distribution varies with the particle shape. When the central primary sphere is larger than the distal sphere $(\delta<0.0)$, the central primary spheres acquire higher charges as shown in Figures $5 \mathrm{a}$ and $5 \mathrm{~b}$. For other cases, the distal spheres tend to have more charge than the central sphere, especially for $\delta>0.0$.

\subsection{The charge distribution and accumulation}

Figure 6 shows the mean charge ratio over particles with different shape factors corresponding to Figure 5. It can be seen that when $\delta<0.0$, the charge ratio is greater on the central spheres than the distal spheres. For the particles of $\delta>0.0$, the larger charge ratio occurs on the distal spheres. It is noticeable that for $\delta=0.65$, the charge ratio of the central sphere is nearly zero. This indicates that, for all typed of particles, the larger primary sphere generally acquires higher net charge than the smaller primary sphere. 


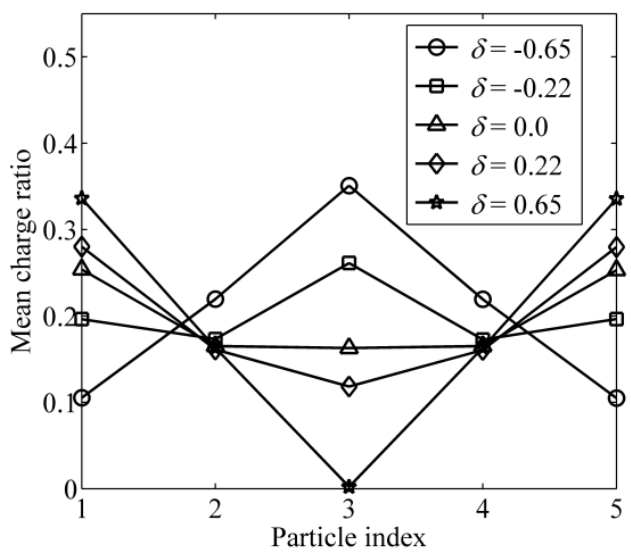

Figure 6 Mean charge ratio of primary spheres over elongated particles with a range of shape factors.

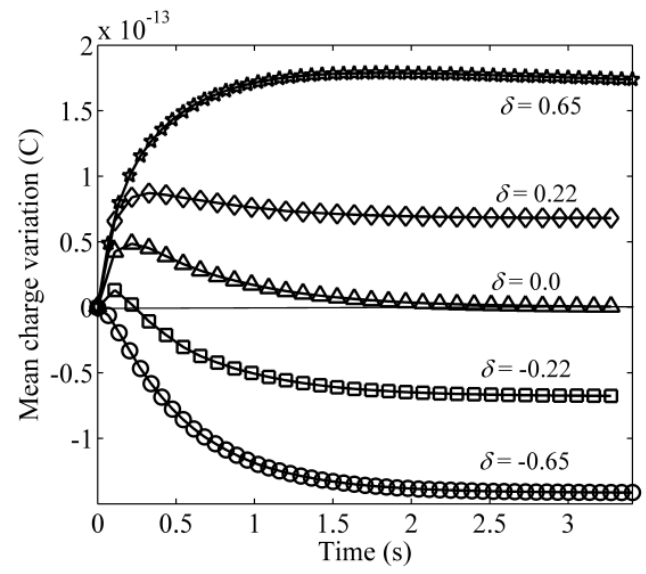

Figure 7 Evolution of mean charge variation over elongated particles with a range of shape factors.

The evolution of the mean charge variation on the elongated particles with the shape factors corresponding those in Figure 6 is shown in Figure 7. For particles with $\delta>0$, the charge variation is positive, i.e., the net charge on the larger distal sphere is higher than that on the smaller central sphere. The charge variation gradually becomes larger and eventually achieves an equilibrium value during the vibration. In addition, the charge variation for $\delta=$ 0.65 is greater than that for $\delta=0.22$. In the case of $\delta=0$, the charge variation is also positive at the earlier stage of the vibration but tends to zero at longer times. When the distal sphere is smaller than the central sphere $(\delta<0)$, the charge variation is negative, which means that the net charge is higher on the central sphere. For both cases of $\delta<0$, the charge variation equilibrates with the value being larger for $\delta=-0.65$. Generally, the larger primary spheres acquire higher net charge, irrespective of their relative position. Furthermore, the charge variation becomes larger during the vibration and eventually achieves an equilibrium value. 


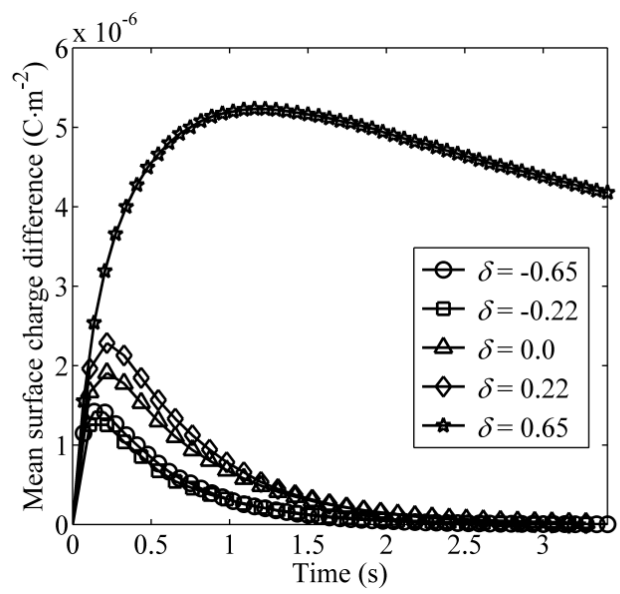

(a)

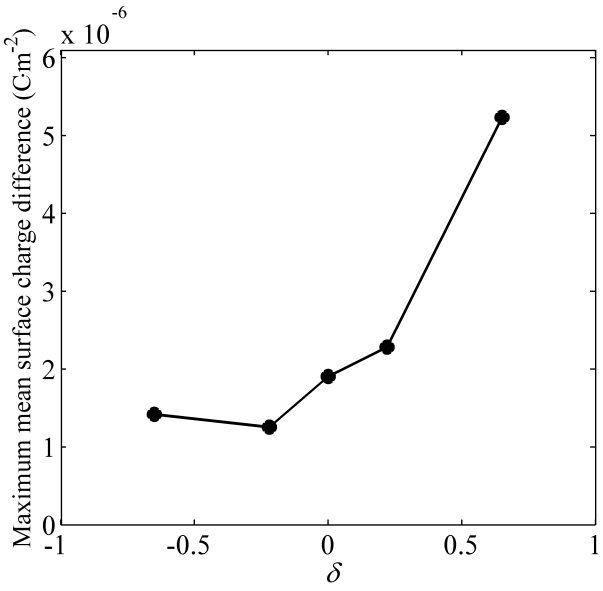

(b)

Figure 8 Surface charge difference over elongated particles with a range of shape factors.

Figure 8 shows the mean surface charge differences for the various shaped particles during the vibrating process. For all particles, the evolution of surface charge difference is similar (Figure 8a). It increases initially and reaches a maximum value. Then it decreases to zero except for the particles with $\delta=0.65$, for which there still is a surface charge difference at the end of the simulation period. In addition, as indicated in Figure 8b, a larger value of $\delta$ leads to a higher surface charge difference during the vibrating process. This indicates that the distal primary sphere has a higher surface charge density than the central sphere at the beginning and the surface charge difference is larger when $\delta$ is larger. Eventually the surface charge density becomes equal between the distal and central spheres except for the particle with $\delta=$ 0.65 .

Figure 9 shows the charge accumulating for various shaped particles during the vibration process. For all cases, the charge gradually accumulates on the particles and eventually achieves an equilibrium value (Figure 9a). However, the equilibrium charge varies with the shape factor (Figure 9b). Specifically, the particles with $\delta=-0.22$ and 0.22 acquire higher equilibrium charges, while the particles with $\delta=-0.65$ possess the lowest equilibrium charge.

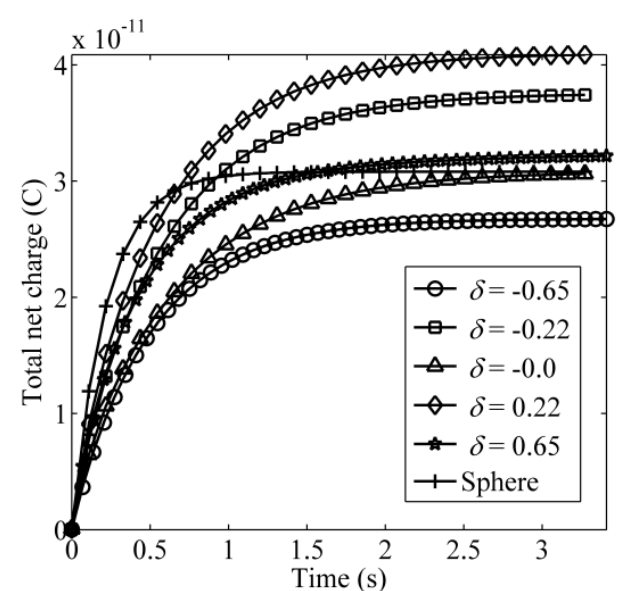

(a)

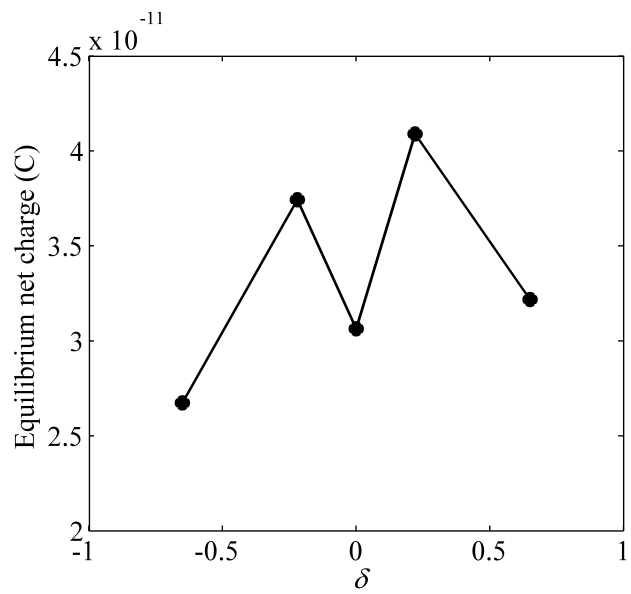

(b)

Figure 9 Charge accumulating processes of various shaped particles. 


\section{Discussion}

In the current study, the charge distribution is obtained on the basis of the net charge on each primary sphere. The transferred charge on each primary sphere depends on the contact frequency that is defined as the total contact number in a unit time. The contact frequency on each primary sphere depends on the orientation and shape of the particle during collisions.

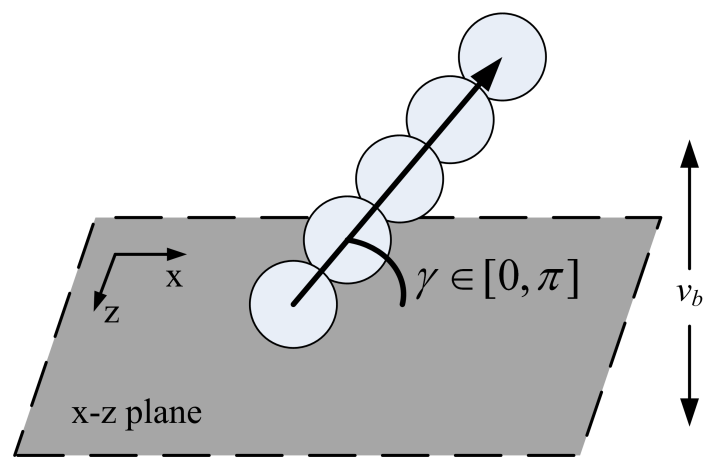

Figure 10 An illustration of the orientation of an elongated particle.

An anisotropy distribution graph is used to explore particle orientation during vibration. The inclination angle between the elongated particle and the lower surface ( $\mathrm{x}-\mathrm{z}$ plane) during the vibration are first calculated as shown in Figure 10. If the $\mathrm{x}$ component of the vector of the elongated particles lies in the $x$ direction, the angle is in the interval of $[0, \pi / 2]$. Otherwise (in $-x$ direction), the angle is in the interval of $[\pi / 2, \pi]$. As the angle is restricted in an interval of $[0, \pi]$, i.e. $0 \leq \gamma \leq \pi$, the interval is divided into 12 sub-intervals. For each particle, its inclination angle to the lower surface should be within one of the sub-intervals. Conversely, the number of particles in each sub-interval can be counted. Consequently, the anisotropy distribution can be defined in a polar coordinate system as 12 triangular sections in the range of 0 to $\pi$. The angle of each triangular section is the angle of each sub-interval and length of each triangular section is the number of particles in each sub-interval. For the vibration process, the number of particles in each section is defined as the mean number of particles in each section over all time instants, which gives the mean anisotropy distribution of the particles. From Figure 4a, it can be seen that all particles tend to align with the lower surface after deposition. To eliminate the effect of this initial orientation of the particles, the calculation of the mean anisotropy distribution is started from the time when all particles are fully activated and start to accumulate charge $(t>0.34 \mathrm{~s})$. This corresponds to the data in Figures 7 and 9 due to the relatively long vibration time $(t>3 \mathrm{~s})$.

Figure 11 shows the mean anisotropy distribution of the particles with different shape factors. It can be seen that similar orientation patterns for all particles are observed. The orientation of most particles concentrates in the triangular sections that are parallel to the lower wall, e.g. $[0, \pi / 12]$ and $[11 \pi / 12, \pi]$. For instance, in Figure 10c $(\delta=0.0)$, most of the particles are within the two sections of $[0, \pi / 12]$ and $[11 \pi / 12, \pi]$. This indicates that most of the particles tend to align perpendicular to the vibration direction. This is because the elongated particles rotate during impacts, especially against the upper and lower walls of the container. Since elongated particles have large aspect ratios, they will rotate towards the impact surface. In addition, the acceleration of the container can force the particles to incline towards the impact surface as shown in Figure 5. 


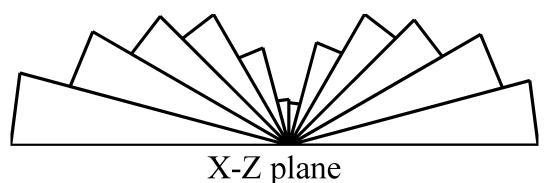

(a) $\delta=-0.65$

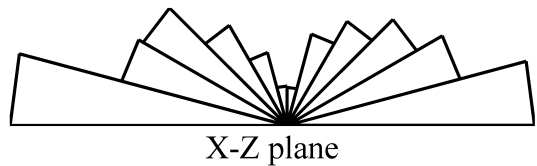

(c) $\delta=0.0$

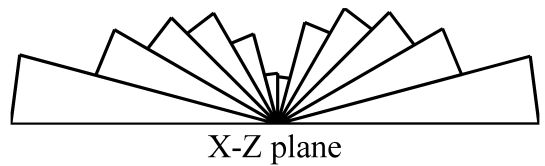

(b) $\delta=-0.22$

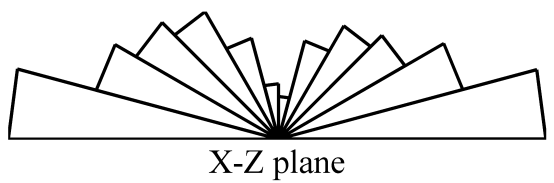

(d) $\delta=0.22$

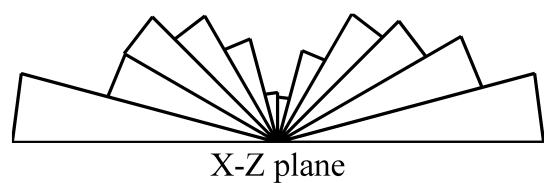

(e) $\delta=0.65$

Figure 11 Particle orientations of various particles.

To evaluate the contact frequency, the total number of contacts on each primary sphere with other surfaces and particles is obtained in the DEM simulations. Then the mean contact frequency of the primary spheres with the index $i$ can be obtained as:

$$
\tau_{i}=\frac{\bar{c}_{i}}{t}
$$

where $\bar{c}_{i}$ is the mean contact number associated with the primary spheres with the same index $i$ and $t$ is the vibration time. Then the contact frequency difference can be defined as the different of mean contact frequency between the distal and central sphere.

Figure 12 shows the mean contact frequency of the distal and central spheres for various particles. depends on the particle shape. For particles with $\delta=-0.65$, the contact frequency of the central sphere is greater than that of the distal sphere. The particle has a larger central primary sphere and tends to align with the lower surface as shown in Figure 11. Thus the central sphere makes more contacts with the lower surface. However, as $\delta$ increases, the contact frequency of the central sphere decreases and the contact frequency of the distal sphere increases, especially when the distal sphere is larger than the central sphere. For example, the contact frequency of the central sphere in the group with $\delta=0.65$ is nearly zero. This indicates that the larger distal spheres (having larger $\delta$ ) contact with the surface and other particles more frequently. 


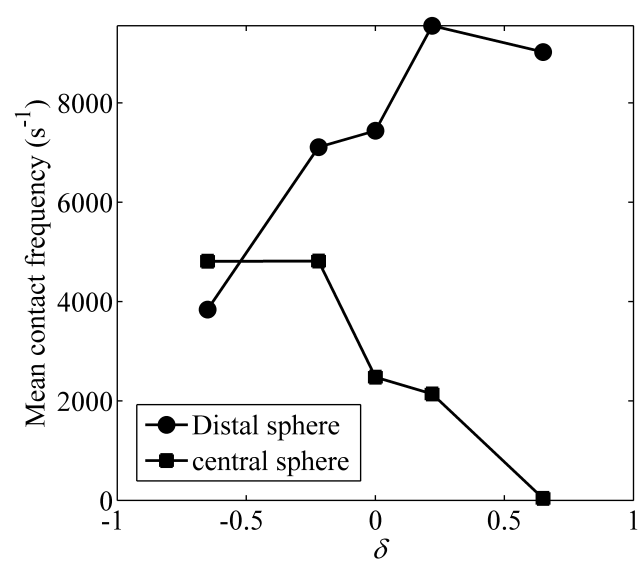

Figure 12 Mean contact frequency during the vibration.

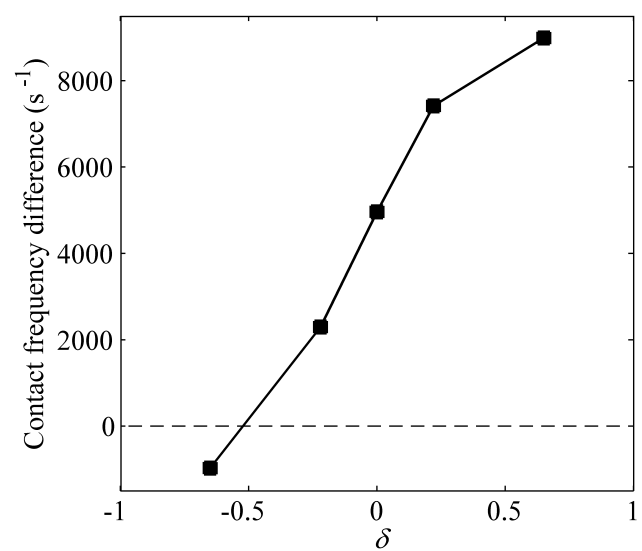

Figure 13 Contact frequency difference for various particles.

Figure 13 shows the contact frequency difference between the distal primary sphere and the central primary sphere for various particles. The value increases as the shape factor increases, which is consistent with Figure 12.

Under similar impact conditions (i.e. the vibration velocity and frequency), spheres with more contacts tend to accumulate a greater net charge. Consequently, the central sphere with a larger size $(\delta<0)$ acquires a larger net charge than the distal sphere as shown in Figure 7. When the distal sphere is larger than the central sphere and makes more contacts with other objects, more net charge is concentrated on the distal sphere. Especially for the group of $\delta=$ 0.65 , the contact frequency of the central sphere is nearly zero, indicating that the central sphere has a small probability of making contact with other objects and is unable to reach its equilibrium state. This eventually leads to a larger surface charge difference as shown in Figure 8 and a lower value of net charge for this group of particles as shown in Figure 9. It is also noticeable that the particles with $\delta=-0.22$ and 0.22 acquire higher equilibrium charges (peaks) in Figure 9b. This is because that for these particles, relatively larger primary spheres are used as shown in Table 1. Therefore, these particles are relatively larger than the rest, which leads to a higher equilibrium charge (Pei et al., 2013).

In the case of the surface charge density, all particles have a larger surface charge density on the distal compared the central sphere as shown in Figure 8a at the earlier stage of the vibration. However, the particles with larger distal spheres have a higher surface charge density at the distal sphere than the groups with smaller distal spheres, as a result of a greater 
contact frequency. According to the contact electrification model (Pei et al., 2013), the net charge on spheres will eventually achieve an equilibrium value and the surface charge density will be the same for spherical particles with different sizes and same material properties. Thus for particles with $\delta \neq 0.65$ as shown in Figure 8a, the distal spheres will eventually have the same surface charge density as the central spheres. For the particles with $\delta=0.65$, the contact rate of the central sphere is sufficiently small that the charge on the central sphere cannot achieve an equilibrium state. Therefore, the surface charge difference of this group is still large at the end of the vibrating process. It is clear that the shape of the particle can affect the charge acquisition and distribution.

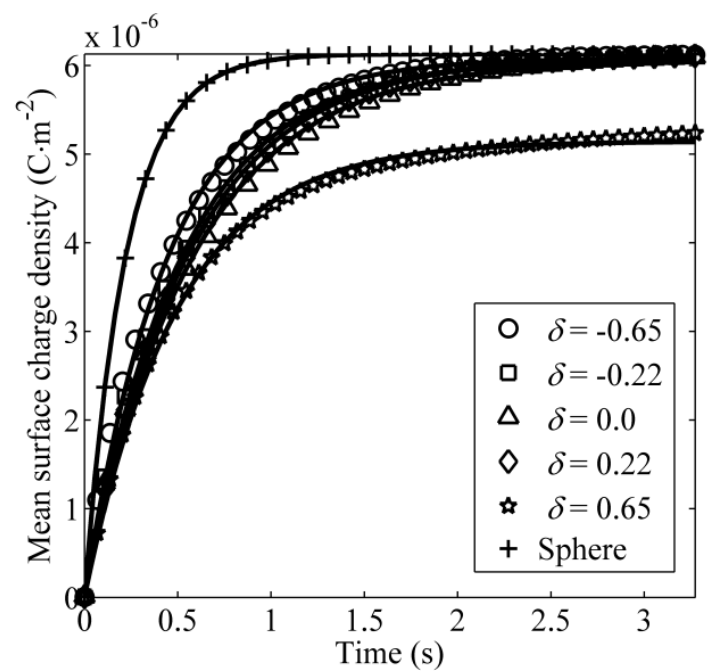

Figure 14 The evolution of mean surface charge density for the various particles.

To study the surface charge density of a particle, a mean surface charge density can be defined as:

$$
\sigma_{p}=\frac{1}{n_{p}} \sum_{s=1}^{n_{p}} \sigma_{s}
$$

where $n_{p}(=5)$ is the number of primary spheres. According to Eq. (9), the transferred charge in each collision is linearly related to the net charge on the particle. Also the net charge can achieve an equilibrium value as shown in Figure 9. Consequently, based on first order kinetics and Eq. (9), the mean surface charge density during vibration can be defined as a function of time thus:

$$
\sigma_{p}=\sigma_{p 0} e^{-k_{c} t}+\sigma_{p \infty}\left(1-e^{-k_{c} t}\right)
$$

where $\sigma_{p 0}$ and $\sigma_{p \infty}$ are the initial mean surface charge density and the equilibrium mean surface charge density and $k_{c}$ is the charging coefficient.

Figure 14 shows the mean surface charge density of various particles during the vibration process. The solid lines are the fitting lines of Eq. (18). It can be seen that the mean surface charge density of various particles gradually increases and eventually achieves a similar equilibrium value except for particles with $\delta=0.65$. The equilibrium value of particles with $\delta$ $=0.65$ are relatively smaller, as the central primary spheres are unable to accumulate charge 
due to the small number of contacts. In summary, the charging process of the elongated particles can be closely represented by the exponential relationship as illustrated by Eq. (18).

Table 3 The charging coefficients for various particles

\begin{tabular}{ll}
\hline$\delta$ & $k_{c}$ \\
\hline-0.65 & 2.23 \\
-0.22 & 1.92 \\
0.0 & 1.73 \\
0.22 & 1.82 \\
0.65 & 2.01 \\
\hline
\end{tabular}

The charging coefficient, $k_{c}$, can be used to determine how fast the charging process achieves equilibrium as given in Table 3. It can be seen that the charging rate coefficient depends on the shape factor. Particles with $\delta=-0.65$ and -0.22 have larger charging rate coefficients than those with $\delta=0$ and 0.22 . This is because the surface charge differences between the particles with $\delta=-0.65$ and -0.22 are smaller and achieve zero faster than those with $\delta=0$ and 0.22 (Figure 8), indicating that the surface charge density of the particles with $\delta=-0.65$ and -0.22 reach steady state faster. In addition, for the case with $\delta=0.65$ the charging rate coefficient is relatively larger due to the lack of charge on the central primary sphere as shown in Figure 8.

It should be noted that the current DEM model is based on the following assumptions: 1) for an elongated particle, the net charge is assumed to be located at the centres of primary spheres; 2) there is no charge relaxation across the particle and dissipation of charges into the environment. Based on these assumptions, non-uniform charge distribution on the particle is observed as shown in Figure 8. These phenomena agree with the experimental observation and theoretical analysis by Matsuyama et al. (2003), in which the non-uniform charge distribution on the surfaces of particles can influence the charge accumulation process and lead to different charging coefficients. The values of transferred charges on particles in the current study are in the range of $10^{-13}$ to $10^{-11} \mathrm{C}$, which is comparable to the typical charge on pharmaceutical particles induced by impact charging (Watanabe et al., 2007). Under realistic conditions, the charge should be distributed on the surface of a particle and may be under relaxation and dissipation, especially for conductors. The contact electrification model employed here ignores the effects of the environmental conditions, such as the humidity and the temperature. In addition, since the net charge is accumulated on the particle, the electrostatic interactions can affect the dynamics of the particle and the subsequent charging process. These effects should be considered further.

In addition, only a small number of particles in a small container are examined in the current study. The orientation of particles is mainly caused by the elongated shape (particle) impacting on a flat surface, which can be considered as one mechanism of contact electrification in the vibration. However, the charging process can vary with the vibration conditions. For instance, when the container vibrates horizontally with a small velocity, the particles can slide on the surface of the container and get charged due to the friction. These phenomena may show different charging behaviours and are worth further investihgation. On the other hand, if a large number of particles are involved in a large scale, the particle-particle contact can become dominant and alter the charge transfer in the particle system. This should also be investigated further, in particular, with the application of high performance computing clusters As DEM is a computer-intensive method. 


\section{Conclusions}

In this study, a discrete element model is developed to study the charge distribution and accumulation on elongated particles in a vibrating container. The particle shape is modelled as a row of primary spheres using the symmetric multi-sphere approach and the charging process is computed by the contact electrification model. Five types of particles are considered.

It is found that, although the charge accumulation process for various cases shows a similar exponential relationship during the vibration and eventually achieves an equilibrium state, the particle shape affects the charge distribution and the charge accumulation on the particles. In terms of the charge distribution, the net charge is greater on the larger primary sphere for each case. Although the surface charge density is always larger on the distal primary sphere, at the earlier stage of the vibration, the surface charge difference between the distal primary sphere and the central sphere increases as the shape factor increases. The surface charge density eventually becomes equal between distal and central spheres except the case of $\delta=$ 0.65 . The central sphere of such a particle cannot achieve an equilibrium surface charge density due to the small number of contacts, which leads to a large surface charge difference between the distal and central sphere even at the end of the vibration. It is also shown that the charging coefficients for the particles with $\delta=0.65$ are different from the case with $\delta=-$ 0.65 , due to the lack of charge on the central sphere. The cases with similar shapes $(\delta=-0.22$ to 0.22 ) have similar charging rates.

This study indicates that particle shape plays an important role in contact electrification. The developed discrete element model can be used to study the charge acquisition, distribution and accumulation process from single particle to the entire particle system. In the future study, the contact electrification model with consideration of charge redistribution and relaxation will be further developed and implemented in DEM..

\section{Acknowledgement}

This project is fully funded by Sanofi (previously sanofi-aventis)

\section{References}

Duff, N., Lacks, D.J., 2008. Particle dynamics simulations of triboelectric charging in granular insulator systems. Journal of Electrostatics 66, 51-57.

Favier, J.F., Abbaspour-Fard, M.H., Kremmer, M., Raji, A.O., 1999. Shape representation of axisymmetrical, non-spherical particles in discrete element simulation using multi-element model particles. Engineering Computations 16, 467-480.

Grzybowski, B.A, Winkleman, A., Wiles, J.A., Brumer, Y., Whitesides, G.M., 2003. Electrostatic self-assembly of macroscopic crystals using contact electrification. Nature materials 2, 241-5.

Haenen, H.T.M., 1976. Experimental investigation of the relationship between generation and decay of charges on dielectrics. Journal of Electrostatics 2, 151-173. 
Hu, G., Hu, Z., Jian, B., Liu, L., Wan, H., 2011. On the determination of the damping coefficient of non-linear spring-dashpot system to model Hertz contact for simulation by Discrete Element Method. Journal of Computers 6, 984-988.

Ireland, P.M., 2010a. Triboelectrification of particulate flows on surfaces: Part I -Experiments. Powder Technology 198, 189-198.

Ireland, P.M., 2010b. Triboelectrification of particulate flows on surfaces: Part II -Mechanisms and models. Powder Technology 198, 199-210.

Ireland, P.M., 2012. Dynamic particle-surface tribocharging: The role of shape and contact mode. Journal of Electrostatics 70, 524-531.

Johnson, K.L., 1985. Contact mechanics. Cambridge University Press, Cambridge.

Kafui, K.D., Thornton, C., Adams, M.J., 2002. Discrete particle-continuum fluid modelling of gas-solid fluidised beds. Chemical Engineering Science 57, 2395-2410.

Kornfeld, M.I., 1976. Frictional electrification. Journal of Physics D: Applied Physics 9, $1183-1192$.

Liu, C., Bard, A.J., 2009. Electrons on dielectrics and contact electrification. Chemical Physics Letters 480, 145-156.

Liu, G., Marshall, J.S., Li, S.Q., Yao, Q., 2010. Discrete-element method for particle capture by a body in an electrostatic field. International Journal for Numerical Methods in Engineering 84, 1589-1612.

Lowell, J., Roseinnes, A.C., 1980. Contact electrification. Advances in Physics 29, 947-1023. Matsusaka, S., Ghadiri, M., Masuda, H., 2000. Electrification of an elastic sphere by repeated impacts on a metal plate. Journal of Physics D: Applied Physics 33, 2311-2319.

Matsusaka, S., Maruyama, H., Matsuyama, T., Ghadiri, M., 2010. Triboelectric charging of powders: A review. Chemical Engineering Science 65, 5781-5807.

Matsuyama, T., Ogu, M., Yamamoto, H., Marijnissen, J.C.M., Scarlett, B., 2003. Impact charging experiments with single particles of hundred micrometre size. Powder Technology $135-136,14-22$.

Matsuyama, T., Yamamoto, H., 1995. Characterizing the electrostatic charging of polymer particles by impact charging experiments. Advanced Powder Technology 6, 211-220.

Mindlin, R.D., Deresiewicz, H., 1953. Elastic spheres in contact under varying oblique forces. Journal of Applied Mechanics-Transactions of The ASME 20, 327-344.

Nwose, E.N., Pei, C., Wu, C.-Y., 2012. Modelling die filling with charged particles using DEM/CFD. Particuology 10, 229-235.

Pei, C., 2013. DEM-CFD analysis of contact electrification and electrostatic interactions during powder handling processes, $\mathrm{PhD}$ thesis. University of Birmingham. 
Pei, C., Wu, C.-Y., Byard, S., England, D., 2010. Numerical analysis of electrostatic effects during powder deposition using DEM/CFD. Journal of Pharmacy and Pharmacology 62, $1454-1455$.

Pei, C., Wu, C.-Y., England, D., Byard, S., Berchtold, H., Adams, M., 2013. Numerical analysis of contact electrification using DEM-CFD. Powder Technology 248, 34-43.

Rezende, C.A, Gouveia, R.F., da Silva, M.A., Galembeck, F., 2009. Detection of charge distributions in insulator surfaces. Journal of physics: Condensed matter: an Institute of Physics journal 21, 263002.

Watanabe, H., Ghadiri, M., Matsuyama, T., Ding, Y.L., Pitt, K.G., Maruyama, H., Matsusaka, S., Masuda, H., 2007. Triboelectrification of pharmaceutical powders by particle impact. International Journal of Pharmaceutics 334, 149-155. 